九州大学学術情報リポジトリ

Kyushu University Institutional Repository

\title{
Trapping of multiple hydrogen atoms in a tungsten monovacancy from first principles
}

Ohsawa, Kazuhito

Research Institute for Applied Mechanics, Kyushu University

Goto, Junya

Interdisciplinary Graduate School of Engineering Science, Kyushu University | Semiconductor Energy Laboratory Co. Ltd.

Yamakami, Masahiro

Interdisciplinary Graduate School of Engineering Science, Kyushu University | Fujitsu Kyushu Network Technologies

Yamaguchi, Masatake

Center for Computational Science and E-Systems, Japan Atomic Energy Agency

他

ht tp://hdl. hand le. net/2324/26364

出版情報: Physical Review B : Condensed Matter and Materials Physics. 82 (18), pp. 184117(1)184117(6), 2010-11-23. American Physical Society

バージョン:

権利関係: (C) 2010 The American Physical Society 


\title{
Trapping of multiple hydrogen atoms in a tungsten monovacancy from first principles
}

\author{
Kazuhito Ohsawa, ${ }^{1, *}$ Junya Goto, ${ }^{2, \dagger}$ Masahiro Yamakami, ${ }^{2, \ddagger}$ Masatake Yamaguchi, ${ }^{3}$ and Masatoshi Yagi ${ }^{1}$ \\ ${ }^{1}$ Research Institute for Applied Mechanics, Kyushu University, Kazuga-koen 6-1, Kasuga-shi, Fukuoka 816-8580, Japan \\ ${ }^{2}$ Interdisciplinary Graduate School of Engineering Science, Kyushu University, Kazuga-koen 6-1, Kasuga-shi, Fukuoka 816-8580, Japan \\ ${ }^{3}$ Center for Computational Science and e-Systems, Japan Atomic Energy Agency, Tokai-mura, Ibaraki 319-1195, Japan
}

(Received 20 May 2010; revised manuscript received 29 October 2010; published 23 November 2010)

\begin{abstract}
The configuration of multiple hydrogen atoms trapped in a tungsten monovacancy is investigated using first-principles calculations. Unlike previous computational studies, which have reported that hydrogen in bcc metal monovacancies occupies octahedral interstitial sites, it is found that the stable sites shift toward tetrahedral interstitial sites as the number of hydrogen atoms increases. As a result, a maximum of twelve hydrogen atoms can become trapped in a tungsten monovacancy.
\end{abstract}

DOI: 10.1103/PhysRevB.82.184117

PACS number(s): $61.82 . \mathrm{Bg}, 61.72 . \mathrm{Bb}, 28.52 . \mathrm{Fa}$

\section{INTRODUCTION}

The interaction between hydrogen $(\mathrm{H})$ and metal is an important subject in the field of metallurgical science. For example, $\mathrm{H}$ absorption and diffusion mechanisms on and in iron $(\mathrm{Fe})$ have been investigated in terms of chemical reactions. ${ }^{1} \mathrm{H}$ retention assists superabundant vacancy formation in many metals ${ }^{2,3}$ and degrades the mechanical properties of metals. $\mathrm{H}$ retention induces embrittlement of iron and steel. ${ }^{4} \mathrm{H}$ bubble formation and blistering reduce the mechanical strength of metals. ${ }^{5}$ In order to examine the $\mathrm{H}$ retention mechanism, a stable configuration of $\mathrm{H}$ atoms trapped in a monovacancy in $\alpha$-Fe was analyzed using firstprinciples calculations. ${ }^{6}$

The interaction between $\mathrm{H}$-isotopes, deuterium and tritium, and plasma facing materials (PFMs) will have a strong impact on the success of the international thermonuclear experimental reactor. The PFMs installed in future fusion reactors are expected to be exposed to high-flux and high-energy plasma particles for long time periods. As a result, many lattice defects will form in the PFMs due to plasma particle collisions. In addition to direct damage, $\mathrm{H}$-isotopes will be retained in vacancy-type lattice defects in the PFMs, a critical problem for fusion reactors. In particular, divertor armor tiles introduced to control the flow of recycling neutrals and impurities back into the core plasma are exposed to the most intense irradiation in magnetic confinement devices. ${ }^{7}$ In such extreme circumstances, tungsten (W) and its alloys show promise as PFMs due to their low $\mathrm{H}$ solubility and excellent thermal and low sputtering properties. ${ }^{8-10}$

This paper elucidates the interaction between $\mathrm{H}$ and $\mathrm{W}$ to reveal fundamental properties of $\mathrm{W}$ that will affect its performance as a PFM. According to previous work, ${ }^{11-13} \mathrm{H}$ is strongly attracted to vacancies in $\mathrm{W}$, one of which can serve as a trapping center for multiple $\mathrm{H}$ atoms. Stable configurations of multiple $\mathrm{H}$ atoms trapped in a monovacancy are investigated using first-principles calculations of lattice relaxation. The binding energy is calculated as a function of the number of $\mathrm{H}$ atoms, which permits estimation of the maximum number of $\mathrm{H}$ atoms that can occupy a $\mathrm{W}$ monovacancy.

One of the purposes of the present work is determining a valid stable configuration of multiple $\mathrm{H}$ atoms trapped in a W monovacancy. It is possible that some metastable configurations and an unexpected ground-state structure exist for the $\mathrm{H}$ atoms. So, a distinct simulation scheme is used. The initial configurations for the $\mathrm{H}$ atoms in the vacancy are randomly generated to perform lattice relaxation and a ground-state structure is selected among some configurations derived from the lattice relaxation.

Zero-point energy (ZPE) for $\mathrm{H}$ is not necessarily negligible in W-H systems, according to our preliminary simulation. ${ }^{14}$ However, ZPEs for $\mathrm{H}$ and $\mathrm{H}$-isotopes depend on the mass number, that is, ZPEs for deuterium and tritium are smaller than that for $\mathrm{H}$. Investigation of equilibrium positions for the $\mathrm{H}$-isotopes in a $\mathrm{W}$ monovacancy is an important subject of the present work. So, two types of binding energies, including and not including ZPE correction for $\mathrm{H}$, will be provided for the sake of simplicity.

\section{METHODOLOGY}

\section{A. Preliminary simulation}

Typical interstitial sites in bcc lattice are tetrahedral and octahedral interstitial sites (T-site and O-site), as shown in Fig. 1(a). T-site is more favorable for interstitial $\mathrm{H}$ than O-site in intrinsic bulk $\mathrm{W} \cdot{ }^{13-15}$ On the other hand, a single $\mathrm{H}$ atom trapped in a W monovacancy is located close to an $\mathrm{O}$-site on the inner surface of the $\mathrm{W}$ monovacancy ${ }^{11}$ as shown in Fig. 1(b), which agrees with our preliminary simulation. ${ }^{14}$ Similarly, multiple $\mathrm{H}$ atoms trapped in a W

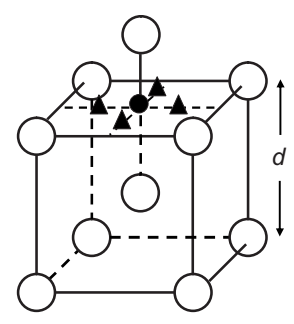

(a) T-site and O-site

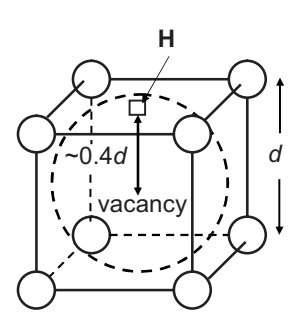

(b) $\mathrm{H}$ atom in vacancy
FIG. 1. (a) Open circles are bcc metal atoms. Closed triangles and closed circles indicate tetrahedral and octahedral interstitial sites, respectively. (b) Schematic view of a single H (open square) trapped in a monovacancy in bcc lattice. 


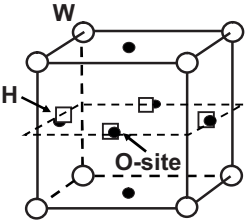

(a) Planar structure

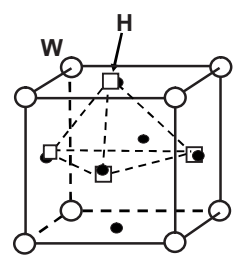

(b) Tetrahedral structure
FIG. 2. Schematic view of (a) planar and (b) tetrahedral structures for four $\mathrm{H}$ atoms in a $\mathrm{W}$ monovacancy. Open circles, open squares, and closed circles indicate $\mathrm{W}, \mathrm{H}$, and $\mathrm{O}$-site, respectively.

monovacancy have been also assumed to be located close to the O-sites. However, we were skeptical about the assumption as follows. As an example, two configurations for four $\mathrm{H}$ atoms in a $\mathrm{W}$ monovacancy are inspected. If the $\mathrm{H}$ atoms are located close to the O-sites on the inner surface of the monovacancy, they become planar or tetrahedral structure, as shown in Fig. 2. H atoms in a $\mathrm{W}$ lattice experience an effective mutual repulsion. ${ }^{13,15,16}$ The tetrahedral structure is flexible to relax the system for the repulsive interaction because the four $\mathrm{H}$ atoms can expand in the vacancy space. So, each $\mathrm{H}$ atom may be largely shifted from the O-site. Actually, the repulsive interaction plays an important role to determine stable configurations for multiple $\mathrm{H}$ atoms trapped in the vacancy, as mentioned later.

\section{B. First-principles calculation}

First-principles calculations based on the densityfunctional theory ${ }^{17}$ are performed using the Vienna $a b$ initio simulation package (VASP) (Refs. 18 and 19) with the generalized gradient approximation ${ }^{20}$ and projected augment wave potentials. ${ }^{21}$ Usually, we employ a bcc supercell composed of 54 lattice points $(3 \times 3 \times 3 \times 2)$ containing a monovacancy and its Brillouin zone with $5 \times 5 \times 5 \mathbf{k}$-point sampling using the Monkhorst-Pack scheme. ${ }^{22}$ The plane-wave energy cutoff is $350 \mathrm{eV}$. Atomic positions and supercell size relaxations are iterated until the force acting on every atom is less than $2 \times 10^{-3} \mathrm{eV} / \AA$. Some cases are checked using a larger bcc supercell composed of 128 lattice points $(4 \times 4 \times 4 \times 2)$ containing a monovacancy with $3 \times 3 \times 3 \mathbf{k}$-point sampling. The energy cutoff is $300 \mathrm{eV}$ for these cases because of computer capacity limits.

Total binding energy for single and multiple $\mathrm{H}$ atoms to a $\mathrm{W}$ monovacancy is defined as

$$
\begin{aligned}
E_{\text {tot }}(\text { conf. })= & E\left[\mathrm{~W}_{n-1} \mathrm{~V}\right]-E\left[\mathrm{~W}_{n-1} \mathrm{VH}_{m}\right] \\
& +m\left(E\left[\mathrm{~W}_{n} \mathrm{H}^{\mathrm{T}}\right]-E\left[\mathrm{~W}_{n}\right]\right),
\end{aligned}
$$

where the function $E$ is the cohesive energy of the supercell. $\mathrm{W}_{n-1} \mathrm{~V}$ is a supercell composed of $(n-1) \mathrm{W}$ and a monovacancy; $\mathrm{W}_{n-1} \mathrm{VH}_{m}$ is that composed of $(n-1) \mathrm{W}$, a monovacancy, and $m \mathrm{H} ; \mathrm{W}_{n} \mathrm{H}^{\mathrm{T}}$ is that composed of $n \mathrm{~W}$ and one $\mathrm{H}$ embedded at a T-site; and $\mathrm{W}_{n}$ is perfect lattice composed of $n \mathrm{~W}$, respectively. The parameter conf. represents the $\mathrm{H}$ configuration in the monovacancy. Therefore, Eq. (1) gives the total energy gain of $m \mathrm{H}$ atoms migrating from distant T-sites and becoming trapped in a monovacancy.

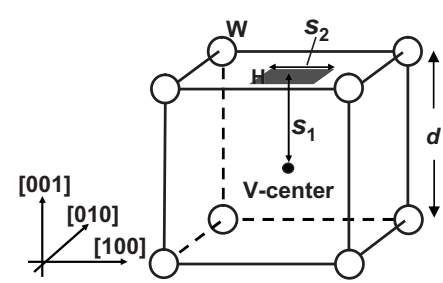

(a) $\boldsymbol{m} \leq 6$

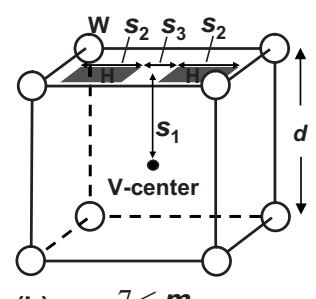

(b) $\quad 7 \leq \boldsymbol{m}$
FIG. 3. Schematic view of initial distribution of $m \mathrm{H}$ atoms in a W monovacancy. Random placement of an $\mathrm{H}$ atom is uniformly distributed within each gray square. (a) Center of square is close to the O-site. (b) Center of square is shifted in the [100] direction. Only gray squares normal to the [001] direction are exhibited but there are equivalent ones on every $\{100\}$ plane.

Average binding energy is defined as

$$
E_{\text {ave }}(\text { conf. })=E_{\text {tot }} / m,
$$

which was used in this and other analyses. ${ }^{11,23}$

Total binding energy including ZPE correction is defined as

$$
E_{z c}(\text { conf. })=E_{t o t}-\mathrm{ZPE}\left(\mathrm{H}_{m}^{\mathrm{V}}\right)+m \mathrm{ZPE}\left(\mathrm{H}^{\mathrm{T}}\right),
$$

where $\mathrm{ZPE}\left(\mathrm{H}_{m}^{\mathrm{V}}\right)$ is the total $\mathrm{ZPE}$ for $m \mathrm{H}$ atoms trapped in a $\mathrm{W}$ monovacancy and $\mathrm{ZPE}\left(\mathrm{H}^{\mathrm{T}}\right)$ is $\mathrm{ZPE}$ for an $\mathrm{H}$ atom embedded at a T-site in intrinsic bulk $\mathrm{W}$ lattice. $\mathrm{ZPE}$ for $\mathrm{H}$ atoms in the $\mathrm{W}$ lattice is calculated by means of harmonic approximation with an adiabatic potential evaluated by first-principles calculations. $^{24}$

\section{Initial configurations for hydrogen atoms in monovacancy}

Investigation of stable configurations of single and multiple $\mathrm{H}$ atoms shows that $\mathrm{H}$ atoms migrate to the inner surface of a monovacancy in metals, ${ }^{6,11,12,23}$ which agrees with the present work. In previous work, the most favorable site for $\mathrm{H}$ was assumed to be the off-vacancy-center position close to the O-site on the inner surface of a W monovacancy. However, this analysis does not require such starting assumptions; the initial configurations for the $\mathrm{H}$ atoms in the vacancy are randomly generated. The $\mathrm{H}$ atoms are initially placed uniformly within one of multiple regions represented by gray squares in Fig. 3. For clarity, only the squares normal to the [001] direction are shown but there are equivalent ones normal to the $\langle 100\rangle$ directions surrounding the monovacancy. The initial configuration has a maximum of one $\mathrm{H}$ atom in each square, leaving some unused when there are fewer $\mathrm{H}$ atoms than squares. The initial distribution is that shown in Fig. 3(a) if $m \leq 6$, and that shown in Fig. 3(b) otherwise, where $m$ is the number of $\mathrm{H}$ atoms trapped in the monovacancy. The parameters $s_{1}, s_{2}$, and $s_{3}$ indicate the distance between the gray square and center of the vacancy, side length of each square, and distance between two squares, respectively. Usually, the values $s_{1}=0.4 d, s_{2}=0.2 d$, and $s_{3}$ $=0.2 d$ are chosen, where $d(\sim 3.17 \AA)$ is the W lattice constant. However, these parameters may be adjusted to account for conditions such as convergence of the lattice relaxation. In our experience, a third of the relaxation processes arrive at 


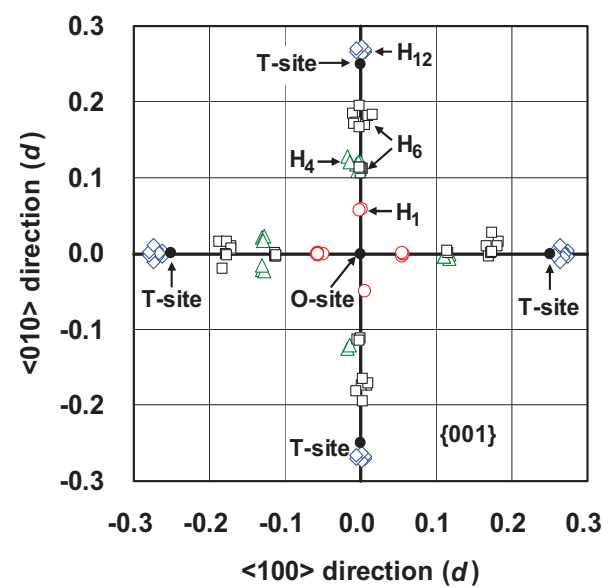

FIG. 4. (Color) Stable positions of $\mathrm{H}$ atoms projected onto the $\{001\}$ plane. Circle, triangle, square, and diamond symbols correspond to the cases where one, four, six, and $12 \mathrm{H}$ atoms are trapped in a monovacancy in a $\mathrm{W}$ lattice, respectively. O-site is located at the center of figure.

a valid ground state while the others arrive at a metastable state or do not finish within the expected number of relaxation steps.

\section{RESULTS}

\section{A. Hydrogen atom in bulk tungsten}

Energetically favorable sites for $\mathrm{H}$ in intrinsic bulk $\mathrm{W}$ are investigated by placing an $\mathrm{H}$ atom at T-site and $\mathrm{O}$-site in the W lattice, as shown in Fig. 1(a). Based on the cohesive energy, T-site is more favorable than $\mathrm{O}$-site by about $0.380 \mathrm{eV}$ for interstitial $\mathrm{H}$, in accordance with the previous work. ${ }^{13,15}$ ZPEs for $\mathrm{H}$ embedded at T-site and $\mathrm{O}$-site, $\mathrm{ZPE}\left(\mathrm{H}^{\mathrm{T}}\right)$ and $\mathrm{ZPE}\left(\mathrm{H}^{\mathrm{O}}\right)$, are estimated to be $0.264 \mathrm{eV}$ and $0.253 \mathrm{eV}$, respectively. Therefore, T-site is still favorable for $\mathrm{H}$ than $\mathrm{O}$-site, if the ZPE correction is taken into account.

\section{B. Ground-state and metastable state configurations of $\mathbf{H}$ atoms in monovacancy}

Stable positions for a single $\mathrm{H}$ in a $\mathrm{W}$ monovacancy are calculated and shown in Fig. 4 projected onto the $\{001\}$

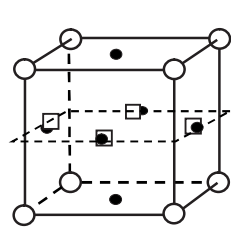

(a) $\begin{aligned} E_{\text {tot }} & =4.126 \\ E_{z c} & =4.586\end{aligned}$ $Z P E=0.594$

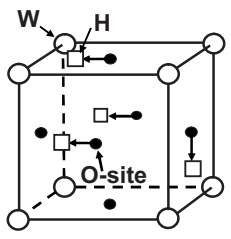

(b) $E_{\text {tot }}=4.368$ $E_{z c}=4.723$

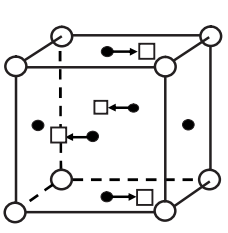

(c) $E_{\text {tot }}=4.358$ $E_{z c}=4.676$ $\mathrm{ZPE}=0.736 \quad(\mathrm{eV})$
FIG. 5. Schematic view of configurations of four $\mathrm{H}$ atoms in a $\mathrm{W}$ monovacancy. Open circles, open squares, and closed circles indicate $\mathrm{W}, \mathrm{H}$, and $\mathrm{O}$-site, respectively. (a) Metastable state; four $\mathrm{H}$ atoms are located close to O-sites on the (001) plane. (b) Ground state; tetrahedral configuration of four $\mathrm{H}$ atoms. (c) State that is nearly degenerate with the ground state.

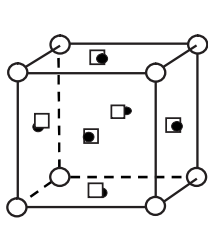

(a) $E_{\text {tot }}=5.456$ $\mathrm{ZPE}=\mathbf{0 . 8 6 1}$ $E_{z c}=6.178$

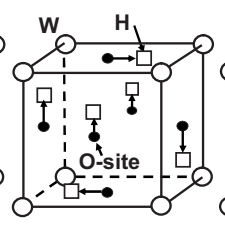

(b) $E_{\text {tot }}=5.886$ $E_{z c}=6.322$ $Z P E=1.146$

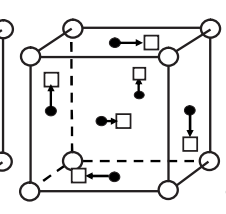

(c) $E_{\text {tot }}=5.886$ $E_{z c}=6.326$ $\mathrm{ZPE}=1.143$

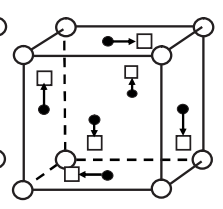

(d) $E_{\text {tot }}=5.888$ $E_{z c}=6.329$ $Z P E=1.140 \quad(e V)$

FIG. 6. Schematic view of configurations of six $\mathrm{H}$ atoms in a vacancy. (a) Metastable state; six $\mathrm{H}$ atoms are located close to the O-sites. (b), (c), and (d) Degenerate ground states; $\mathrm{H}$ atoms are considerably shifted from the $\mathrm{O}$-sites.

plane. Single H occupies a location close to the O-site, which corresponds to the work of Liu et al. very well. ${ }^{11}$ However, the positions are slightly shifted in the $\langle 100\rangle$ or $\langle 010\rangle$ direction by about $0.05 d$, as represented by circle symbols in Fig. 4.

Stable configurations for four, six, eight, nine, and $12 \mathrm{H}$ atoms trapped in a $\mathrm{W}$ monovacancy are explained in detail and shown in Figs. 5-9, respectively. Binding energies, $E_{\text {tot }}$ and $E_{z c}$, are defined in Eqs. (1) and (3), and the ZPE in these figures means the total zero-point energy for multiple $\mathrm{H}$ atoms trapped in a W monovacancy, where energy unit is electron volt $(\mathrm{eV})$. Open circles, open squares, and closed circles indicate $\mathrm{W}, \mathrm{H}$, and $\mathrm{O}$-site, respectively.

The planer configuration for four $\mathrm{H}$ atoms shown in Fig. 5(a) is not a ground-state structure. Instead, the tetrahedral configuration of $\mathrm{H}$ atoms in Fig. 5(b) is a ground state and that in Fig. 5(c) has a nearly degenerate binding energy. The $\mathrm{ZPE}$ for $\mathrm{H}$ atoms is not negligible compared with the binding energy. However, the $\mathrm{H}$ configuration of Fig. 5(b) is still ground state, if ZPE correction is taken into account.

Many types of stable and metastable configurations can be found for the case of six $\mathrm{H}$ atoms. As in previous work, binding energy is calculated by assuming that each $\mathrm{H}$ atom starts close to the O-site in a W monovacancy, as shown in Fig. 6(a). In this case, the binding energy is estimated to be $E_{\text {tot }}\left(6 \mathrm{H}^{\mathrm{O}}\right)=5.456 \mathrm{eV}$. However, the $\mathrm{H}$ configurations shown in Figs. 6(b)-6(d) are found to be more stable, with binding energy estimated to be $E_{t o t}(6 \mathrm{H})=5.886-5.888 \mathrm{eV}$. By taking into account finite temperature, these energetically
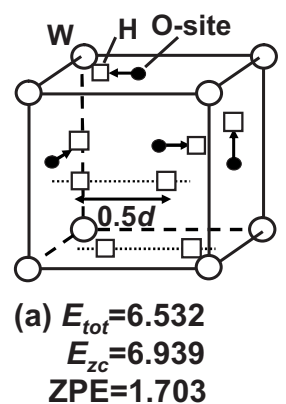

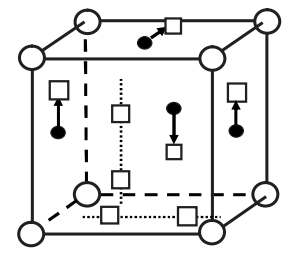

(b) $E_{\text {tot }}=6.514$ $E_{z c}=6.942$ ZPE $=1.682$
FIG. 7. Schematic view of configurations of eight $\mathrm{H}$ atoms in a W monovacancy. Open circles, open squares, and closed circles indicate $\mathrm{W}, \mathrm{H}$, and $\mathrm{O}$-site, respectively. (a) and (b) are nearly degenerate states. Ground state changes when ZPE correction is taken into account. 


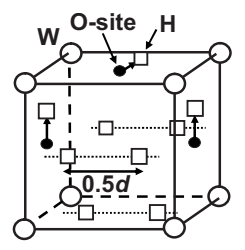

(a) $\begin{aligned} E_{\text {tot }} & =6.638 \\ E_{z c} & =7.041\end{aligned}$ $\mathrm{ZPE}=1.972$

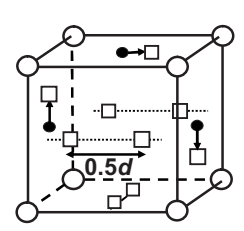

(b) $\begin{aligned} E_{\text {tot }} & =6.492 \\ E_{z c} & =6.901\end{aligned}$ $Z P E=1.965$

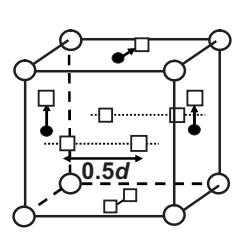

(c) $E_{\text {tot }}=6.298$ $E_{z c}=6.713$ $\mathrm{ZPE}=1.959 \quad(\mathrm{eV})$
FIG. 8. Schematic view of configurations of nine $\mathrm{H}$ atoms in a W monovacancy. (a) Ground state. (b) and (c) Metastable states. H atoms are located close to T-sites.

equivalent configurations can be regarded as degenerate ground states. The six $\mathrm{H}$ atoms in these configurations are not located close to the $\mathrm{O}$-sites but considerably shifted in the $\langle 100\rangle$ direction by a large displacement of $0.1 d-0.2 d$. Four $\mathrm{H}$ atoms shift by about $0.18 d$ and the remaining two shift by about $0.12 d$ from the O-site, as shown in Figs. 4 and 6(b)-6(d). The three configurations of six $\mathrm{H}$ atoms are still degenerate ground states, when ZPE correction is taken into account.

As mentioned above and shown in Fig. 4, stable positions for the $\mathrm{H}$ atoms in a $\mathrm{W}$ monovacancy gradually move away from the O-site in the $\langle 100\rangle$ direction as the number of trapped $\mathrm{H}$ atoms increases. Here, we assume that the most favorable site for $\mathrm{H}$ is the T-site in the high $\mathrm{H}$ density limit. Thus, the stable configurations of more than six $\mathrm{H}$ atoms in a $\mathrm{W}$ monovacancy are sought by assuming that $\mathrm{H}$ atoms are located close to the T-sites. So, the initial distribution of $\mathrm{H}$ atoms in Fig. 3(b) is used for lattice relaxation.

Configurations for eight $\mathrm{H}$ atoms trapped in a $\mathrm{W}$ monovacancy are shown in Fig. 7. The binding energies for these configurations are nearly degenerate. The ground-state structure changes by taking account of ZPE correction, which is the only case in the present simulations.

Configurations for nine $\mathrm{H}$ atoms in a $\mathrm{W}$ monovacancy are shown in Fig. 8. The nine $\mathrm{H}$ atoms are located close to the $\mathrm{T}$-sites. The ZPE for each $\mathrm{H}$ configuration is estimated to be about $2 \mathrm{eV}$, which is very large. However, the difference among the ZPEs is so slight as to be negligible. As a result, ZPE corrections do not play an essential role to determine ground state.

Stable and metastable configurations for the case of 12 trapped $\mathrm{H}$ atoms are shown in Fig. 9. Two $\mathrm{H}$ atoms close to the T-sites are arranged in the $\langle 100\rangle$ direction on every $\{100\}$

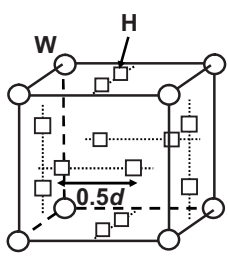

(a) $\begin{aligned} E_{\text {tot }} & =7.391 \\ E_{z c} & =7.863\end{aligned}$ $\mathrm{ZPE}=\mathbf{2 . 6 9 2}$

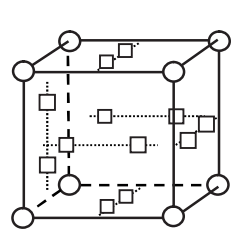

(b) $\begin{aligned} E_{\text {tot }} & =6.759 \\ E_{z c} & =7.286\end{aligned}$

$Z P E=2.638$

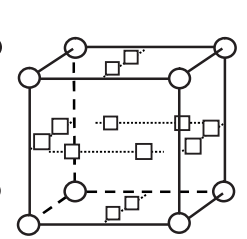

(c) $E_{\text {tot }}=6.096$

$E_{z c}=6.660$ $Z P E=2.601 \quad(e V)$
FIG. 9. Schematic view of configurations of $12 \mathrm{H}$ atoms in a monovacancy. (a) Ground state. (b) and (c) Metastable states. H atoms are located close to T-sites.

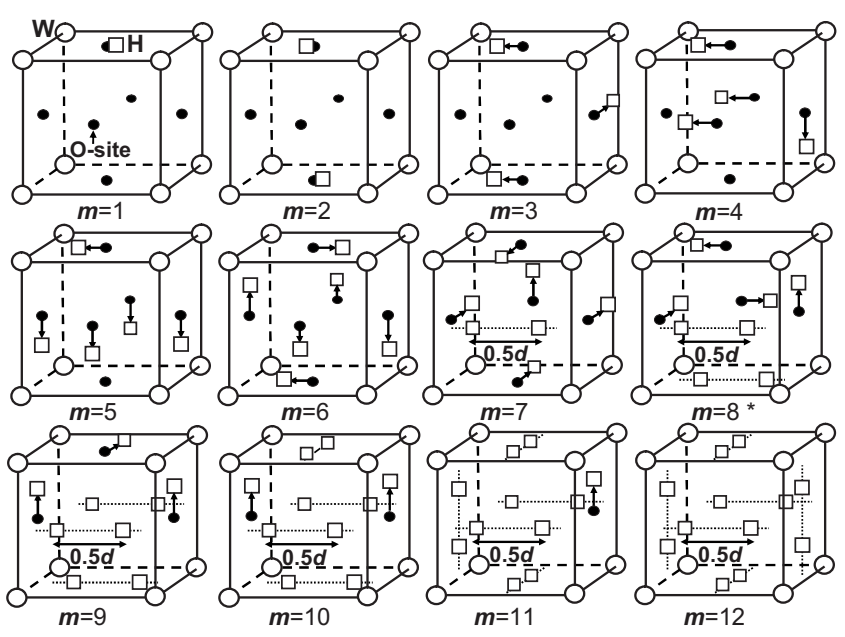

FIG. 10. Schematic view of ground-state structures of $\mathrm{H}$ atoms in a W monovacancy. Open circles, open squares, and closed circles indicate $\mathrm{W}, \mathrm{H}$, and $\mathrm{O}$-site, respectively. $m$ is the number of $\mathrm{H}$ atoms in the vacancy. ${ }^{*}$ refer to Fig. 7 in case of $m=8$.

plane. The distance between the two $\mathrm{H}$ atoms is approximately $d / 2(\sim 1.6 \AA)$, much longer than an $\mathrm{H}_{2}$ molecule bond of length $0.75 \AA$. Therefore, these $\mathrm{H}$ pairs are not $\mathrm{H}_{2}$ molecules. The distance between $\mathrm{H}$ atoms is maximized because $\mathrm{H}$ atoms in a $\mathrm{W}$ lattice experience an effective mutual repulsion. ${ }^{13,15,16}$ Thus, the configuration shown in Fig. 9(a) is expected to be the lowest-energy state.

Figure 10 shows ground-state structures of single and

TABLE I. Total binding energy $E_{t o t}$, average binding energy $E_{\text {avr }}$, binding energy including ZPE correction $E_{z c}$, and total ZPE $(\mathrm{eV})$ for ground-state structures of single and multiple $\mathrm{H}$ atoms, where $m$ is the number of $\mathrm{H}$ atoms trapped in a $\mathrm{W}$ monovacancy. ZPEs (eV) for $\mathrm{H}$ embedded at a T-site and O-site in intrinsic bulk W are also listed, as below. * refer to Fig. 7 in case of $m=8$.

\begin{tabular}{lcccc}
\hline \hline$m$ & $\begin{array}{c}E_{\text {tot }} \\
(\mathrm{eV})\end{array}$ & $\begin{array}{c}E_{\text {avr }} \\
(\mathrm{eV})\end{array}$ & $\begin{array}{c}E_{\text {zc }} \\
(\mathrm{eV})\end{array}$ & $\begin{array}{c}\mathrm{ZPE} \\
(\mathrm{eV})\end{array}$ \\
\hline 1 & 1.182 & 1.182 & 1.318 & 0.128 \\
2 & 2.353 & 1.176 & 2.626 & 0.254 \\
3 & 3.423 & 1.141 & 3.708 & 0.506 \\
4 & 4.368 & 1.092 & 4.723 & 0.700 \\
5 & 5.256 & 1.051 & 5.652 & 0.922 \\
6 & 5.888 & 0.981 & 6.329 & 1.140 \\
7 & 6.173 & 0.881 & 6.597 & 1.423 \\
$8(\mathrm{a})^{*}$ & 6.532 & 0.816 & 6.939 & 1.703 \\
$8(\mathrm{~b})^{*}$ & 6.514 & 0.814 & 6.942 & 1.682 \\
9 & 6.638 & 0.737 & 7.041 & 1.972 \\
10 & 6.979 & 0.697 & 7.407 & 2.210 \\
11 & 7.101 & 0.645 & 7.550 & 2.452 \\
12 & 7.391 & 0.615 & 7.863 & 2.692 \\
13 & 6.541 & 0.503 & 7.082 & 2.889 \\
14 & 7.365 & 0.526 & 7.819 & 3.239 \\
$\mathrm{ZPE}\left(\mathrm{H}^{\mathrm{T}}\right)$ & 0.264 & & & \\
$\mathrm{ZPE}\left(\mathrm{H}^{\mathrm{O}}\right)$ & 0.253 & & & \\
\hline \hline
\end{tabular}




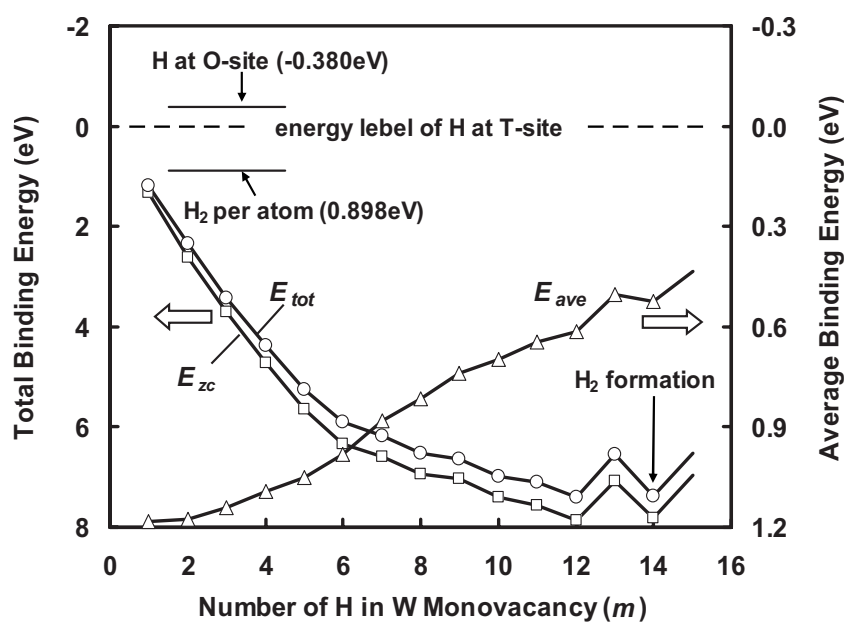

FIG. 11. Total (circles) and average (triangles) binding energy as function of $\mathrm{H}$ atom number trapped in a $\mathrm{W}$ monovacancy. Total binding energy including ZPE correction (squares) is also expressed. Zero energy level corresponds to energy of $\mathrm{H}$ atom embedded at a T-site.

multiple $\mathrm{H}$ atoms in a W monovacancy, where $m$ is the number of $\mathrm{H}$ atoms. Each $\mathrm{H}$ atom slightly or largely shifts from an $\mathrm{O}$-site in the $\langle 100\rangle$ direction. In case of $m \geq 7$, most $\mathrm{H}$ atoms are located close to T-sites. The binding energy and ZPE for every ground state are listed in Table I.

\section{Maximum number of $\mathbf{H}$ atoms in monovacancy}

The number of $\mathrm{H}$ atoms that a $\mathrm{W}$ monovacancy can accommodate was expected to be quite large because there are $24 \mathrm{~T}$-sites on the inner surface of a monovacancy in a bcc lattice. Figure 11 shows the dependence of total binding energy on the $\mathrm{H}$ atom number. The ground-state structures of the multiple $\mathrm{H}$ atoms are evaluated by comparing the energy of simulated stable and metastable configurations. The total binding energy gradually increases as the number of $\mathrm{H}$ atoms increases to 12 but ceases to increase once the number exceeds 12. Additional $\mathrm{H}$ atoms move out of the vacancy and become trapped at T-sites outside the vacancy during the relaxation process. Occasionally, the additional $\mathrm{H}$ atoms remain inside the vacancy as isolated $\mathrm{H}$ atoms, lowering the total binding energy. As the $\mathrm{H}$ atom number increases above twelve, the binding energy does not decrease monotonically; the formation of a $\mathrm{H}_{2}$ molecule inside the vacancy contributes to an increase in the binding energy. In particular, the total binding energy for $14 \mathrm{H}$ atoms with the $\mathrm{H}_{2}$ molecule formation is estimated to be $7.365 \mathrm{eV}$, which is almost the same as that for $12 \mathrm{H}$ atoms of the ground state $(7.391 \mathrm{eV})$. However, no more than one $\mathrm{H}_{2}$ molecule is observed inside the monovacancy at one time. As a result, $12 \mathrm{H}$ atoms can be accommodated in a W monovacancy.

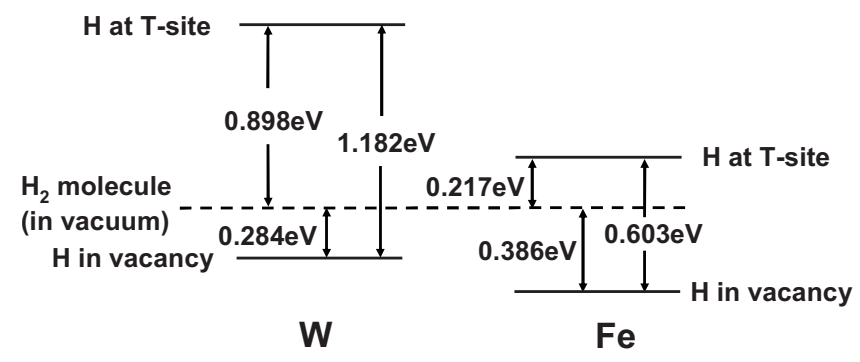

FIG. 12. Energy landscape of $\mathrm{W}$ and Fe lattice representing total energy gain of $\mathrm{H}$ trapping in a monovacancy. ZPE is ignored in figure.

\section{DISCUSSION}

To explain why a monovacancy in $\mathrm{W}$ can accommodate so many $\mathrm{H}$ atoms, we refer to the energy landscape of $\mathrm{H}$ in $\mathrm{W}$ and Fe shown in Fig. 12. One of the prominent properties of $\mathrm{W}$ is an extremely high chemical potential with $\mathrm{H}(0.898$ $\mathrm{eV})$. Thus, $\mathrm{H}$ is not usually retained in bulk W. However, there is a significant capacity for the $\mathrm{H}$ density to increase in an irradiation environment such as a fusion reactor. The energy gain is very large when an $\mathrm{H}$ embedded at a T-site migrates to a monovacancy in the $\mathrm{W}$ lattice $(1.182 \mathrm{eV})$. This large energy gain, which traps a large number of $\mathrm{H}$ in the monovacancy, is not necessarily realized in other bcc metals; the energy gain of $\mathrm{H}$ migration in an Fe lattice is only 0.603 $\mathrm{eV}{ }^{14}$

ZPE for multiple $\mathrm{H}$ atoms trapped in a W monovacancy is not necessarily negligible compared with binding energy. However, the ZPE is not essential to determine ground-state and metastable state structures because the effect of total binding energy $E_{\text {tot }}$ is usually larger than that of ZPE correction. ZPE approximately depends only on the number of $\mathrm{H}$ atoms in the monovacancy but on the $\mathrm{H}$ configuration. As a result, the dependence of $E_{t o t}$ and $E_{z c}$ on the trapped $\mathrm{H}$ atom number in a W monovacancy are substantially same, as shown in Fig. 11. However, the ground-state structure for eight $\mathrm{H}$ atoms in the monovacancy changes by the effect of ZPE correction, as shown in Fig. 7. This is only an isolated case but attracts our attention.

These simulations showed that $\mathrm{H}$ atoms move from the $\mathrm{O}$-sites to more stable positions close to the T-sites as they increase in number, as shown in Fig. 4. This phenomenon may be related to the fact that a single $\mathrm{H}$ preferentially occupies a T-site in a perfect bcc lattice.

\section{ACKNOWLEDGMENTS}

This work was supported by a Grant-in-Aid for Scientific Research (Grant No. 20560616) from the Japan Society for the Promotion of Science. We would like to express to Aaron Froese our gratitude for his devoted support. 
*ohsawa@riam.kyushu-u.ac.jp

†Present address: Semiconductor Energy Laboratory Co. Ltd., 398 Hase, Atsugi-shi, Kanagawa 243-0036, Japan.

\$Present address: Fujitsu Kyushu Network Technologies, 2-2-1 Momochihama, Sawara-ku, Fukuoka-shi, Fukuoka 814-8588, Japan.

${ }^{1}$ D. E. Jiang and E. A. Carter, Phys. Rev. B 70, 064102 (2004).

${ }^{2}$ Y. Fukai and N. Okuma, Phys. Rev. Lett. 73, 1640 (1994).

${ }^{3}$ Y. Fukai, Phys. Scr. T103, 11 (2003).

${ }^{4}$ J. P. Hirth, Metall. Trans. A 11, 861 (1980).

${ }^{5}$ J. B. Condon and T. Schober, J. Nucl. Mater. 207, 1 (1993).

${ }^{6}$ Y. Tateyama and T. Ohno, Phys. Rev. B 67, 174105 (2003).

${ }^{7}$ P. C. Stangeby, The Plasma Boundary of Magnetic Fusion Devices (Taylor \& Francis, London, 2000), p. 212.

${ }^{8}$ W. M. Shu, E. Wakai, and T. Yamanishi, Nucl. Fusion 47, 201 (2007).

${ }^{9}$ M. Tokitani, M. Miyamoto, K. Tokunaga, T. Fujiwara, N. Yoshida, S. Masuzaki, N. Ashikawa, T. Morisaki, M. Shoji, A. Komori, LHD Experimental Group, S. Nagata, and B. Tsuchiya, J. Nucl. Mater. 363-365, 443 (2007).

${ }^{10}$ N. Yoshida, J. Nucl. Mater. 266-269, 197 (1999).

${ }^{11}$ Y.-L. Liu, Y. Zhang, H.-B. Zhou, G.-H. Lu, F. Liu, and G.-N.
Luo, Phys. Rev. B 79, 172103 (2009).

${ }^{12}$ C. S. Becquart and C. Domain, J. Nucl. Mater. 386-388, 109 (2009).

${ }^{13}$ Y.-L. Liu, Y. Zhang, G.-N. Luo, and G.-H. Lu, J. Nucl. Mater. 390-391, 1032 (2009).

${ }^{14}$ M. Yamakami, M.S. thesis, Kyushu University, 2009; M. Yamakami, M. Yagi, and K. Ohsawa, Rep. Res. Inst. Appl. Mech. (Kyushu Univ.) 137, 81 (2009).

${ }^{15}$ J. Xu and J. Zhao, Nucl. Instrum. Methods Phys. Res. B 267, 3170 (2009).

${ }^{16}$ K. O. E. Henriksson, K. Nordlund, A. Krasheninnikov, and J. Keinonen, Appl. Phys. Lett. 87, 163113 (2005).

${ }^{17}$ P. Hohenberg and W. Kohn, Phys. Rev. 136, B864 (1964).

${ }^{18}$ G. Kresse and J. Hafner, Phys. Rev. B 47, 558 (1993).

${ }^{19}$ G. Kresse and J. Furthmüller, Phys. Rev. B 54, 11169 (1996).

${ }^{20}$ J. P. Perdew and Y. Wang, Phys. Rev. B 45, 13244 (1992).

${ }^{21}$ P. E. Blöchl, Phys. Rev. B 50, 17953 (1994).

${ }^{22}$ H. J. Monkhorst and J. D. Pack, Phys. Rev. B 13, 5188 (1976).

${ }^{23}$ G. Lu and E. Kaxiras, Phys. Rev. Lett. 94, 155501 (2005).

${ }^{24}$ R. Matsumoto, Y. Inoue, S. Taketomi, and N. Miyazaki, Scr. Mater. 60, 555 (2009). 